\title{
Serum potential biomarkers according to sputum inflammatory cell profiles in adult asthmatics
}

\author{
Gyu-Young Hur ${ }^{1}$, Young-Min Ye ${ }^{2}$, Eunmi Yang ${ }^{2}$, and Hae-Sim Park ${ }^{2}$
}

${ }^{1}$ Department of Internal Medicine, Korea University College of Medicine, Seoul; ${ }^{2}$ Department of Allergy and Clinical Immunology, Ajou University School of Medicine, Suwon, Korea

Received: March 10, 2019

Revised : May 14, 2019

Accepted: May 31, 2019

\section{Correspondence to}

Hae-Sim Park, M.D.

Department of Allergy and Clinical Immunology, Ajou University School of Medicine, 164 World cup-ro, Yeongtong-gu, Suwon 16499, Korea

Tel: +82-31-219-5196

Fax: +82-31-219-4265

E-mail: hspark@ajou.ac.kr

https://orcid.org/0000-0003-

2614-0303
Background/Aims: Asthma is not a single disease but, rather, a heterogeneous inflammatory disorder with various pathogenic mechanisms. We analyzed the associations between the cellular profile of sputum and the serum levels of inflammatory mediators/cytokines in a cohort of adult asthmatics.

Methods: We recruited 421 adult asthmatic patients. All subjects were classified into four groups according to their sputum cellular profiles: G1, eosinophilic; G2, mixed granulocytic; $\mathrm{G}_{3}$, neutrophilic; and $\mathrm{G}_{4}$, paucigranulocytic. Serum levels of cytokines and mediators including periostin, eosinophil-derived neurotoxin (EDN), S10oA9, and folliculin were quantified.

Results: Among 421 patients, G1 accounted for 149 (35.4\%), G2 for 71 (16.9\%), G3 for 155 (36.8\%), and $G_{4}$ for 46 (10.9\%). Serum periostin and EDN levels were significantly higher in $\mathrm{G} 1(p=0.004$, and $p=0.031)$ than in the others. Serum S100A9 levels were elevated in $\mathrm{G}_{2}$ and $\mathrm{G}_{3}(p=0.008)$. Serum folliculin levels differed significantly among the four groups, with the highest level in $\mathrm{G}_{4}(p=0.042)$. To identify G1 from G1 plus G2 groups, the optimal serum cut-off levels were 1.71 $\mathrm{ng} / \mathrm{mL}$ for periostin, and $1.61 \mathrm{ng} / \mathrm{mL}$ for EDN. When these two parameters were combined, the sensitivity was $76.0 \%$ and the specificity was $64.3 \%$ (area under the curve, $0.701 ; p=0.004)$.

Conclusions: The serum periostin and EDN levels may be used as predictors to discriminate the eosinophilic asthma group from patients having eosinophilic or mixed granulocytic asthma, and the serum folliculin level is significantly elevated in patients with paucigranulocytic asthma compared to those with different inflammatory cell profile.

Keywords: Asthma; Biomarkers; Eosinophils; Neutrophils

\section{INTRODUCTION}

Asthma is a heterogeneous airway disease with complex pathological mechanisms. Many attempts have been made to define asthma subtypes technically, based on clinical, physiologic and pathologic heterogeneities [1]. Th2 mechanisms and eosinophils have been identified as the main target with regard to asthmatic inflammation in severe asthma [2]; however, the pathogenic mech- anisms of non-eosinophilic asthma have not yet been fully characterized.

Recently, the presence of dual-positive Th2/Th17 cells has been observed in the blood [3] and bronchoalveolar lavage fluid [4] of asthmatic patients. Based on these observations, a new classification of Th2-predominant, Th2/Th17-predominant, and Th2/Th17-low asthma has been suggested. The interleukin-1 $\beta$ (IL-1 $\beta$ ) pathway was revealed as a critical pathway for defining the Th2/ 
Th17-predominant or the Th2/Th17-low asthma groups, suggesting that different mechanisms are involved in the pathogenesis of neutrophilic asthma [5]. Th2-dominant asthma is characterized by elevated numbers of eosinophils [2] and levels of biomarkers such as periostin and eosinophil-derived neurotoxin (EDN), which are closely associated with eosinophilic airway inflammation [6]. Th2/Th17-predominant asthma may drive the mixed granulocytic asthma group, where the lowest lung function is observed [7]. Th2/Th17-low asthma traditionally includes both neutrophilic and paucigranulocytic asthma groups, in which neutrophils and epithelial cells play major roles.

The S100 protein family is the most abundant in neutrophils and macrophages, among which $\mathrm{S}_{100 \mathrm{~A} 9}$ is reported to have roles in neutrophilic asthma [8]. Additionally, folliculin is a protein derived from airway epithelial cells. Its production is increased by epithelial cell activation in asthmatic patients [9].

The results of testing procedures for induced sputum cellular profiles are often affected by multiple complex factors making them unstable and inconsistent [10]. Timing of tests and previous use of corticosteroid treatment may lead to different results. There are difficulties when implementing and interpreting sputum cell counts, so counting blood eosinophils has been revisited during clinical trials [11]. However, in cases of severe asthma, counting blood eosinophils is not sufficiently reliable to characterize airway inflammation, because the patient has been exposed to recurrent infections and frequent systemic corticosteroid therapy [12]. Therefore, there is a need to find another consistent and reliable disease marker to reflect the character and degree of airway inflammation in an adult asthma cohort.

In this study, we measured the serum levels of inflammatory mediators and cytokines in adult asthmatics and analyze their associations with the cellular profile of sputum. Furthermore, we investigated the ability of serologic markers to distinguish the various cellular profiles of the sputum of adult asthmatics.

\section{METHODS}

\section{Study subjects}

Among 1,133 adult asthmatics [13] in Ajou University
Medical Center (Suwon, Korea), 421 with available sputum profiles were enrolled in this study. All patients were consistent with the diagnosis of bronchial asthma and had been treated with maintenance medications, including medium-to-high doses of inhaled corticosteroids. The patients had a history of respiratory symptoms and evidence of airflow limitation such as airway reversibility and airway hyperreactivity [14]. Their demographic data were collected, including disease duration, past history, history of aspirin-exacerbated respiratory disease (AERD), and smoking status. Smoking status was classified into current and not current smokers (previous or never smoker).

The Institutional Review Board of Ajou University Hospital approved this study (AJIRB-GEN-SMP-13-108). Signed written consent and agreement to participate in the study were obtained from each subject.

\section{Assessment of clinical parameters}

All participants underwent skin prick tests with common inhalant allergens to define their atopy status. Total eosinophil counts (TEC) in peripheral blood were obtained. Total serum immunoglobulin E (IgE) and eosinophilic cationic protein (ECP) levels were measured using the ImmunoCAP system (Thermo Fisher Scientific Inc., Waltham, MA, USA). Chest/paranasal sinus X-ray and/or computed tomography and rhinoscopy were completed during enrollment into this study. Lung function tests including spirometry with bronchodilator, and methacholine challenge testing were conducted to diagnose bronchial asthma during the initial visit.

Severe asthma was diagnosed as asthma that required treatment with high doses of inhaled corticosteroids plus a second controller and/or systemic corticosteroids and that "remained uncontrolled" despite this therapy. Uncontrolled asthma was defined as the presence of one or more of the following: (1) poor asthma control, asthma control test <20; (2) frequent severe exacerbations, defined as two or more bursts of systemic corticosteroids during the previous year; (3) serious exacerbations, defined as at least one hospitalization during the previous year; (4) airflow limitation, \% predicted value of post-bronchodilator forced expiratory volume in 1 second $\left(\mathrm{FEV}_{1}\right)<80 \%$ [15]. Patients with a previous diagnosis of chronic obstructive pulmonary 
disease (COPD) and/or asthma-COPD overlap by clinicians were excluded from this study.

\section{Sputum collection and classification}

Sputum induction with hypertonic saline (4.5\%) was performed as previously described [16]. The subjects did not cease their currently used inhaled corticosteroid. Sputum samples were collected in a plastic container and dispersed using dithiothreitol for sputum cell count assessment and inflammatory subtype determination. Inflammatory cells were counted as a percentage of total sputum cells. Neutrophilic cut-off values were age-dependent and defined as previously described [17]. Neutrophilic or mixed granulocytic groups were determined according to the following age groups: neutrophil percentage $(<20$ years old), $75.57 \%$ or greater; neutrophil percentage (20 to 40 years old), $61.61 \%$ or greater; neutrophil percentage (40 to 60 years old), $63.25 \%$ or greater; and neutrophil percentage ( $>60$ years old), $67.25 \%$ or greater $[17]$.

The study groups were determined as follows: group 1 (G1, eosinophilic group) was defined as having sputum eosinophilia (eosinophil percentage $\geq 3 \%$ in sputum); group 2 (G2, mixed granulocytic group) was defined as having both sputum eosinophilia and neutrophilia; group 3 ( $\mathrm{G}_{3}$, neutrophilic group) was defined as having sputum neutrophilia; and group 4 (G4, paucigranulocytic group) was defined as having neither sputum eosinophilia nor neutrophilia

\section{Measurements of serum inflammatory mediators}

We searched for serum inflammatory mediators reflecting the sputum cellular profiles of asthmatic patients from published literature reviews. We selected four candidate mediators; periostin, EDN, S100A9, and folliculin. We performed enzyme-linked immunosorbent assay (ELISA) for the candidate mediators. Each mediator was assayed using subjects' frozen sera following ELISA kits according to the manufacturer's instructions. The ELISA kits used were used as follows: measuring serum levels of periostin (Shino-Test, Kanagawa, Japan), EDN (MBL International, Woburn, MA, USA), S10oA9 (R\&D Systems, Minneapolis, MN, USA), and folliculin (CUSA Biotech, Wuhan, China).

\section{Statistical analysis}

All serum levels of cytokines in the study participants were log-transformed before conducting statistical analysis to establish a normal distribution. Data analysis was performed using SPSS version 22.0 (IBM Co., Armonk, NY, USA). The values are expressed as means \pm standard deviation or frequencies (\%). One-way analysis of variance (ANOVA) was used to compare differences among the four groups, whereas Student's $t$ test was used to compare two groups. Statistical correlations were analyzed using Pearson's correlation coefficient.

To evaluate factors associated with $\mathrm{G} 1$ among G1 plus G2, a binary logistic regression was performed. All of the values were adjusted for age, gender, and smoking status. Receiver operating characteristic (ROC) curves were used to evaluate the validity of serum periostin and EDN levels for discriminating G1 from G1 plus G2, and the area under the curve (AUC) with a 95\% confidence interval (CI) was computed. Sensitivity and specificity were calculated according to the identified optimal cut-offs. Values of $p$ of $<0.05$ were considered indicative of statistical significance. GraphPad Prism version 8.o (GraphPad, La Jolla, CA, USA) was used to create graphs.

\section{RESULTS}

\section{Clinical findings of the study subjects according to sputum cell profile}

Among the 421 subjects, 149 were G1 (35.4\%), 71 were G2 (16.9\%), 155 were $\mathrm{G}_{3}(36.8 \%)$ and 46 were $\mathrm{G}_{4}(10.9 \%)$. Females were predominant in $\mathrm{G}_{2}(64.8 \%)$ and $\mathrm{G}_{3}(68.4 \%)$, compared to $\mathrm{G}_{1}(55.0 \%)$ and $\mathrm{G}_{4}(58.7 \%)$ (Table 1$)$. No significant differences were observed in age and duration of asthma among the four study groups. The prevalence of atopy, AERD, current smokers, chronic rhinosinusitis, and nasal polyps were similar among the four groups. TEC was significantly elevated in $\mathrm{G}_{1}$ and $\mathrm{G}_{2}(p=0.005$ by ANOVA), compared to G3 or G4. Serum total IgE and ECP levels did not differ significantly among the four groups. Lung functions including $\mathrm{FEV}_{1}$ (\% predicted) and $\mathrm{PC}_{20}(\mathrm{mg} / \mathrm{mL}$, the methacholine concentration causing a $20 \%$ fall in $\mathrm{FEV}_{1}$ ) did not differ significantly among the four groups, but the mean $\mathrm{FEV}_{1}$ levels tended to be lower in G2 subjects, and the prevalence of severe asthma tended to be higher in G2 subjects. 
Table 1. Comparison of clinical findings of asthma patients classified according to the results of sputum inflammatory cell profiles

\begin{tabular}{|c|c|c|c|c|c|}
\hline Characteristic & $\mathrm{Gl}(\mathrm{n}=149)$ & $\mathrm{G} 2(\mathrm{n}=71)$ & $\mathrm{G}_{3}(\mathrm{n}=155)$ & $\mathrm{G}_{4}(\mathrm{n}=46)$ & $p$ value \\
\hline Age, yr & $43.59 \pm 12.99$ & $43 \cdot 32 \pm 13 \cdot 77$ & $45.07 \pm 14.32$ & $44.25 \pm 13.91$ & 0.727 \\
\hline Disease duration, yr & $5.61 \pm 5 \cdot 51$ & $5.94 \pm 6.32$ & $6.73 \pm 7.71$ & $5.7 \pm 5.28$ & 0.675 \\
\hline Female sex, \% & $55 \cdot 0$ & 64.8 & 68.4 & 58.7 & 0.102 \\
\hline Atopy, \% & 51.0 & $53 \cdot 5$ & 55.2 & $45 \cdot 7$ & 0.690 \\
\hline $\mathrm{AERD}, \%$ & 46.3 & $43 \cdot 7$ & 38.1 & 50.0 & 0.373 \\
\hline Current smokers, \% & 29.9 & 48.9 & 33.0 & 51.7 & 0.056 \\
\hline Chronic rhinosinusitis, \% & 82.2 & 80.0 & 80.6 & 76.9 & 0.936 \\
\hline Nasal polyps, \% & 38.0 & $23 \cdot 4$ & $25 \cdot 7$ & 20.0 & 0.107 \\
\hline $\mathrm{TEC}, / \mu \mathrm{L}$ & $580.2 \pm 605.73$ & $511.65 \pm 1058.5$ & $332.85 \pm 328.01$ & $374.34 \pm 538.13$ & 0.005 \\
\hline Total IgE, kU/L & $461.47 \pm 931.2$ & $365.32 \pm 442.2$ & $396.87 \pm 805.09$ & $263.48 \pm 344.07$ & 0.470 \\
\hline $\mathrm{ECP}, \mu \mathrm{g} / \mathrm{L}$ & $39.6 \pm 42.6$ & $32.61 \pm 39.88$ & $35.04 \pm 36.45$ & $27 \cdot 77 \pm 31.51$ & 0.419 \\
\hline Sputum eosinophils, \% & $64.76 \pm 26.86$ & $15.69 \pm 18.62$ & $0.3 \pm 0.68$ & $0.15 \pm 0.6$ & $\leq 0.001$ \\
\hline Sputum neutrophils, \% & $22.57 \pm 19.11$ & $79.68 \pm 9.35$ & $89.47 \pm 8.23$ & $31.87 \pm 19.42$ & $\leq 0.001$ \\
\hline $\mathrm{FEV}_{1}, \%$ predicted & $86.6 \pm 20.7$ & $78.74 \pm 24.66$ & $86.73 \pm 21.10$ & $84.90 \pm 23.07$ & 0.060 \\
\hline $\mathrm{PC}_{20}, \mathrm{mg} / \mathrm{mL}$ & $7.99 \pm 9.57$ & $5.77 \pm 7.98$ & $8.51 \pm 9.61$ & $5 \cdot 72 \pm 7 \cdot 71$ & 0.191 \\
\hline Severe asthma, \% & 16.8 & 28.2 & $15 \cdot 5$ & 21.7 & 0.118 \\
\hline
\end{tabular}

Values are presented as mean $\pm \mathrm{SD}$.

G1, eosinophilic asthma group; G2, mixed granulocytic asthma group; $\mathrm{G}_{3}$, neutrophilic asthma group; G4, paucigranulocytic asthma group; AERD, aspirin-exacerbated respiratory disease; TEC, total eosinophil count; IgE, immunoglobulin E; ECP, eosinophilic cationic protein; $\mathrm{FEV}_{1}$, forced expiratory volume in 1 second; $\mathrm{PC}_{20}$, the methacholine concentration causing a $20 \%$ fall in $\mathrm{FEV}_{1}$.

Table 2. Serum biomarkers classified with the results of sputum cell profiles

\begin{tabular}{lccccc}
\hline Variable & $\mathrm{G} 1(\mathrm{n}=149)$ & $\mathrm{G} 2(\mathrm{n}=71)$ & $\mathrm{G} 3(\mathrm{n}=155)$ & $\mathrm{G}_{4}(\mathrm{n}=46)$ & $p$ value \\
\hline Log periostin, $\mathrm{ng} / \mathrm{mL}$ & $1.92 \pm 0.17$ & $1.80 \pm 0.26$ & $1.84 \pm 0.19$ & $1.80 \pm 0.28$ & 0.004 \\
Log EDN, ng/mL & $1.81 \pm 0.25$ & $1.64 \pm 0.32$ & $1.71 \pm 0.25$ & $1.69 \pm 0.22$ & 0.031 \\
Log ECP, ug/L & $1.35 \pm 0.54$ & $1.21 \pm 0.62$ & $1.31 \pm 0.53$ & $1.26 \pm 0.45$ & 0.461 \\
Log S10oA9, pg/mL & $0.45 \pm 0.62$ & $0.85 \pm 0.72$ & $0.83 \pm 0.70$ & $0.39 \pm 0.59$ & 0.008 \\
Log folliculin, pg/mL & $1.41 \pm 0.53$ & $1.10 \pm 0.70$ & $1.40 \pm 0.62$ & $1.48 \pm 0.65$ & 0.042 \\
\hline
\end{tabular}

Values are presented as mean $\pm \mathrm{SD}$.

$\mathrm{G}$, eosinophilic asthma group; $\mathrm{G} 2$, mixed granulocytic asthma group; $\mathrm{G}_{3}$, neutrophilic asthma group; $\mathrm{G}_{4}$, paucigranulocytic asthma group; EDN, eosinophil-derived neurotoxin; ECP, eosinophilic cationic protein.

\section{Comparison of serum inflammatory biomarkers among the four groups}

Serum levels of inflammatory mediators were log-transformed, including periostin, EDN, ECP, folliculin, and S1ooA9, to correct their skewed distributions. Table 2 lists the serum inflammatory parameters and the values according to each group. Serum periostin and EDN levels were significantly higher in $\mathrm{G}_{1}(p=0.004$ and $p=$ 0.031, by ANOVA). Fig. 1 compares four serum inflammatory mediator concentrations within the four groups: serum periostin level was the highest in $\mathrm{G}_{1}$ (G1 vs. G2, $p$ $=0.002 ; G_{1}$ vs. G3, $p=0.004 ;$ and G1 vs. G4, $p=0.007$ ) (Fig. 1A). Serum EDN levels were more significantly elevated in $\mathrm{G}_{1}$ than $\mathrm{G}_{2}$ and $\mathrm{G}_{3}$ (G1 vs. $\mathrm{G} 2, p=0.010$; and $\mathrm{G} 1$ vs. 


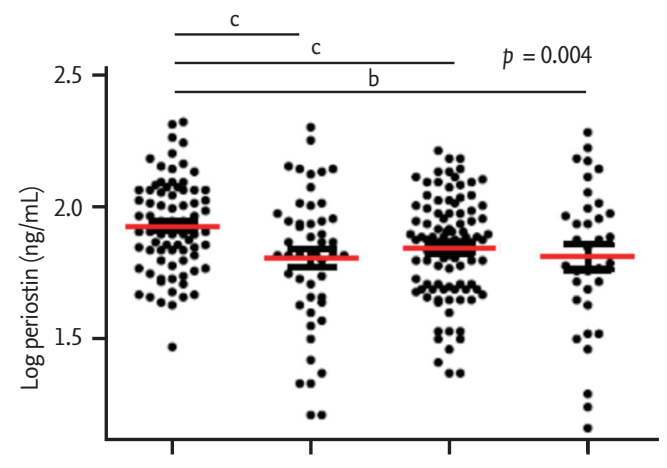

Gl

G2

G3

G4

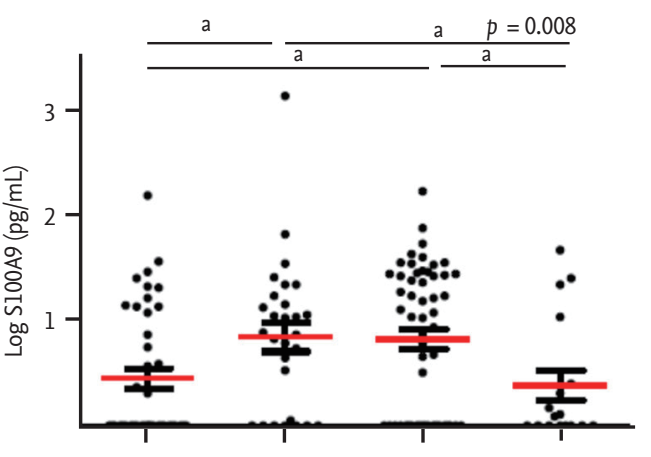

$\mathrm{Gl}$

G2

G3

G4

D
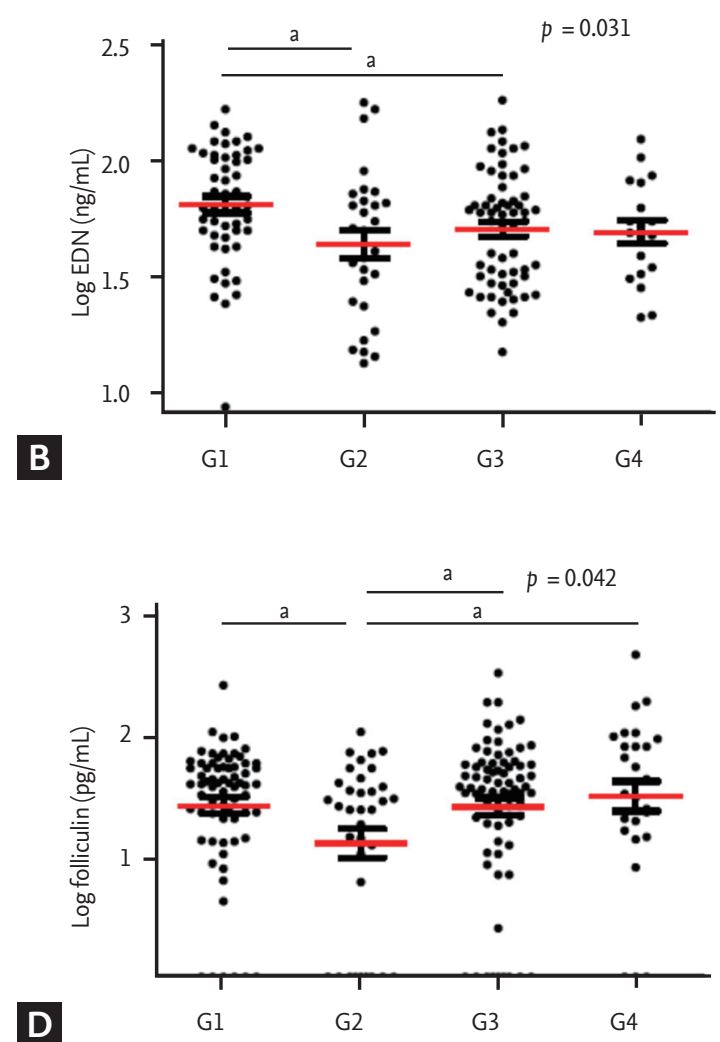

Figure 1. Serum cytokine levels of (A) periostin, (B) EDN, (C) S10oA9, and (D) folliculin according to sputum inflammatory cell profile. Individual data were compared by analysis of variance, and group data were compared by Student's $t$ test. G1, eosinophilic ( $n=149)$; $G 2$, mixed ( $n=71) ; G_{3}$ neutrophilic $(n=155) ; G 4$, paucigranulocytic group ( $\left.n=46\right)$. EDN, eosinophil-derived neurotoxin. ${ }^{\mathrm{a}} \mathrm{p}<0.05,{ }^{\mathrm{b}} \mathrm{p}<0.01,{ }^{\mathrm{c}} \mathrm{p}<0.005$, by Student's $t$ test.

$\mathrm{G}_{3}, p=0.030$ ) (Fig. 1B). No significant differences were observed in serum ECP levels among the groups.

Serum S10oA9 levels differed significantly among the four groups ( $p=0.008$, by ANOVA) (Table 2). The levels in G2 and G3, the two groups with increased neutrophil counts in sputum were significantly higher than those of the other two groups (G1 vs. G2, $p=0.017$; $\mathrm{G}_{1}$ vs. $\mathrm{G}_{3}, p$ $=0.008 ; G_{2}$ vs. $G_{4}, p=0.031 ; G_{3}$ vs. $G_{4}, p=0.022$ ) (Fig. 1 C). Serum folliculin levels differed significantly among the four groups ( $p=0.042$ by ANOVA) (Table 2$)$. They were significantly lower in G2 than in the other three groups, with the highest levels found in $\mathrm{G}_{4}$ (G1 vs. G2, $p=0.013$; G2 vs. $G_{3}, p=0.025 ; G_{2}$ vs. $G_{4}, p=0.029$ ) (Fig. 1D).

\section{Comparison of serum inflammatory biomarkers among eosinophil-dominant groups}

Because both G1 and G2 were eosinophil-dominant groups, we compared serum mediators to find factors that might differentiate between these two groups. Serum periostin and EDN levels were more significantly elevated in $\mathrm{G} 1$ than in $\mathrm{G} 2(p=0.002$ and $p=0.010)$ (Fig. 1), whereas S10oAg levels were significantly lower in G1 than in G2 ( $p=0.017$ ) (Fig. $1 C$ ). Serum EDN levels were positively correlated with serum periostin $(r=0.288, p=$ 0.011 ) (Fig. 2A) and ECP $(r=0.243, p=0.038)$ (Fig. 2B) levels in G1 plus G2. Serum EDN levels had a stronger correlation with TEC than periostin $(r=0.335, p=0.003$ for TEC, and $r=0.217, p=0.013$ for periostin, data not shown).

The diagnostic values of the serum EDN and periostin levels were evaluated to discriminate $\mathrm{G} 1$ from G1 plus G2 using a ROC curve (Fig. $3 \mathrm{~A}$ ), and we selected the appropriate cut-off values. Using the cut-off value for periostin $(\geq 1.74 \mathrm{ng} / \mathrm{mL}$ ), the sensitivity and specificity were $87.5 \%$ and $42.3 \%$ with 0.696 (95\% CI, 0.563 to $0.829 ; p=0.007$ ) of the AUC value. When we applied the cut-off value of EDN $(\geq 1.61 \mathrm{ng} / \mathrm{mL}$ ), the sensitivity and specificity were $85.0 \%$ 


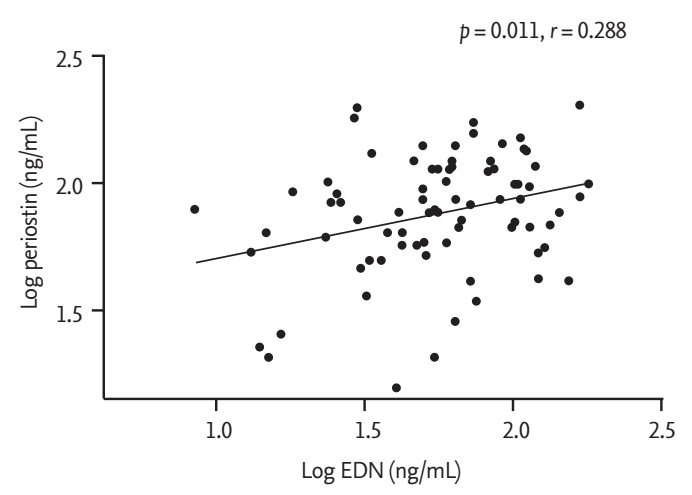

A

Figure 2. Correlations of the level of eosinophil-derived neurotoxin (EDN) with that of (A) periostin, and (B) eosinophilic cationic protein (ECP) in G1 plus G2 as determined by calculating the Pearson correlation coefficient.

A

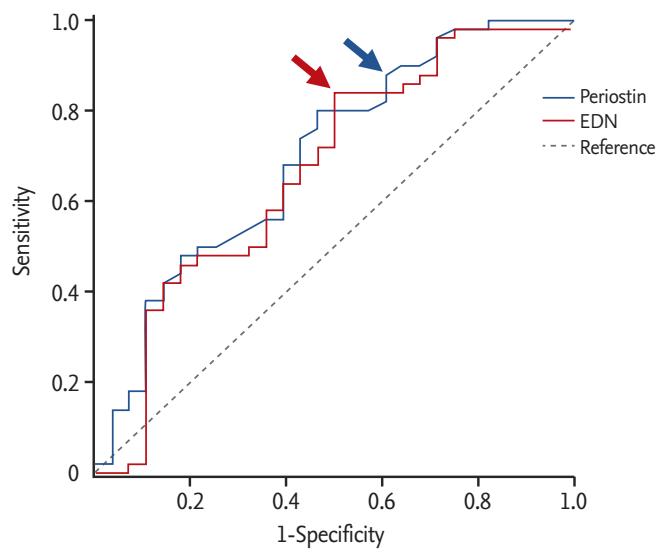

B

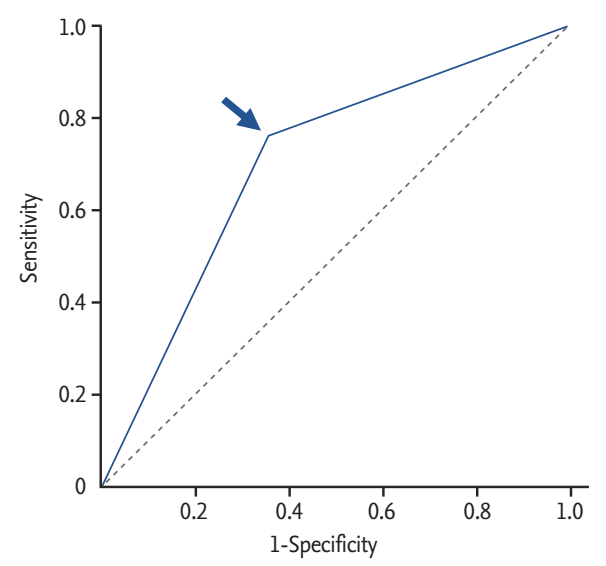

Figure 3. Receiver operating characteristic curves for the serum levels of periostin and eosinophil-derived neurotoxin (EDN) (A), as well as the combined value (B) to discriminate the optimal cut-offs for G1 from G1 plus G2. Arrows indicate the optimal value for periostin (blue arrow in A), EDN (red arrow in A), and the combined value (blue arrow in B; periostin $\geq 1.74 \mathrm{ng} / \mathrm{mL}$ and $\mathrm{EDN} \geq 1.61 \mathrm{ng} / \mathrm{mL})$.

Table 3. Sensitivity, specificity, and the values of the area under the curve with $95 \%$ confidence interval

\begin{tabular}{|c|c|c|c|c|}
\hline Cut-offs & Sensitivity & Specificity & $\operatorname{AUC}(95 \% \mathrm{CI})$ & $p$ value \\
\hline Log periostin $\geq 1.71 \mathrm{ng} / \mathrm{mL}$ & 0.875 & 0.423 & $0.696(0.563-0.829)$ & 0.007 \\
\hline $\log \mathrm{EDN} \geq 1.61 \mathrm{ng} / \mathrm{mL}$ & 0.850 & 0.462 & $0.655(0.514-0.796)$ & 0.035 \\
\hline Log periostin $\geq 1.74 \mathrm{ng} / \mathrm{mL}$ and $\log \mathrm{EDN} \geq 1.61 \mathrm{ng} / \mathrm{mL}$ & 0.760 & 0.643 & $0.701(0.577-0.826)$ & $0.004^{\mathrm{a}}$ \\
\hline
\end{tabular}

A $p$ values are calculated using logistic regression analysis.

AUC, area under the curve; CI, confidence interval; EDN, eosinophil-derived neurotoxin.

${ }^{a}$ Values are adjusted by age, sex, and smoking status.

and $46.2 \%$ with 0.655 (95\% CI, 0.514 to $0.796 ; p=0.035$ ) of the AUC value. When both parameters were combined, the AUC value increased to 0.701 (95\% CI, 0.577 to $0.826 ; p$ $=0.004$ ), whereas the sensitivity and specificity increased to $76.0 \%$ and $64.3 \%$, respectively (Table 3 and Fig. 3 ). The $p$ value of combined parameters was obtained using logistic regression after the adjustment by age, gender, and smoking status. Serum folliculin and S10oAg levels were excluded for the ROC curve analysis, as their $p$ values were marginal ( $p=0.038$ and $p=0.048$, respectively). 


\section{DISCUSSION}

Asthma classification using inflammatory cell profiles of sputum has been useful for characterizing inflammatory subtypes and predicting treatment responses [18]. Sputum eosinophilia is a useful tool for defining the phenotype of eosinophilic asthma and is a good predictor of the patient's response to inhaled steroids [19]. However, some asthmatic patients have both sputum neutrophilia and eosinophilia; therefore, they present as mixed granulocytic asthma. The phenotypes of remaining non-eosinophilic subjects are still heterogeneous and can be further classified based on the presence of neutrophilia, as neutrophilic only, and as paucigranulocytic asthma [18,20]. Many efforts have been made to fully characterize the different subtypes of adult asthmatics based on sputum cell profiles, but they are not yet fully understood.

In this study, we assigned adult asthmatic patients into four groups based on their sputum cell profiles: $\mathrm{G} 1$, only eosinophilic; G2, mixed granulocytic, both eosinophilic and neutrophilic; $\mathrm{G}_{3}$, only neutrophilic; and $\mathrm{G}_{4}$, paucigranulocytic asthma. This is an easier diagnostic method in real practice, compared to flow cytometry techniques. $\mathrm{G}_{1}$ and $\mathrm{G}_{3}$ were the predominant groups, followed by G2 and G4. There have been variable differences in the prevalence of sputum cell profiles between studies, which are mainly due to differences in study populations, and the cut-offs of sputum eosinophil/neutrophil counts used. We used the same cut-off values as those of a previous study: $>3 \%$ eosinophils for eosinophilic asthma, and age-dependent cut-offs for neutrophils as neutrophilic asthma [21]. However, other studies have used different cut-off points for neutrophilic asthma: $>61 \%$ of sputum neutrophils [22] and a different cut-off point for eosinophilic asthma, > 1.1\% of sputum eosinophils [18]. Another Korean study [23] used different cut-off values for sputum neutrophilia ( $\geq 40 \%)$, leading to different results from the ones that were observed in this study. We used the recommendations of a recent report stating that age-specific reference values should be used as a cut-off point for neutrophilic inflammation in clinical studies, as airway neutrophilia is related to age in adults [17]. Overall, the variable classification results in sputum inflammatory cell profiles are likely due to various cut-off values that were applied to different groups.

We compared clinical parameters among four different groups in this study. G1 and G2 have sputum eosinophilia typified by significantly high levels of peripheral blood eosinophils with no observable differences between these groups. However, other type-2 response markers such as total IgE and ECP levels did not differ significantly among the four different groups. Lung function tests revealed that $\mathrm{G} 2$ tended toward lower $\mathrm{FEV}_{1} \%$ and $\mathrm{PC}_{20}$ methacholine levels compared to the other three groups, finding that are in agreement with other studies reporting lower $\mathrm{FEV}_{1} \% / \mathrm{PC}_{20}$ methacholine levels or poor asthma controls in G2 [7,18,24]. Previous research has shown a significant association between asthma severity and mixed granulocytic asthma [25]. Microassay screening of sputum samples has demonstrated that mediators such as brain-derived neutrophilic factor (BDNF), and IL-1 $\beta$, have a stronger association with neutrophils. These were elevated in patients with mixed granulocytic asthma [7]. These findings suggest that mixed granulocytic asthma is associated with reduced lung function and severe asthma in which additional The inflammatory components, such as BDNF and IL-1 $\beta$, could contribute to a poor outcome.

Serum levels of periostin and EDN were the highest in G1 compared to the other three groups, while no significant difference was noted in ECP level among the groups. Additionally, serum EDN level was positively correlated with periostin level, suggesting that both serum periostin and EDN levels could be potential serum biomarkers to predict the phenotype of eosinophil-dominant group among asthmatic patients with sputum eosinophilia. Serum EDN levels have been suggested as a marker for monitoring the functional status of eosinophils in various allergic diseases including asthma, atopic dermatitis, allergic rhinitis, and eosinophilic esophagitis [26]. In childhood asthma, serum EDN levels were reported to be clinically relevant as a marker for predicting asthma severity and monitoring and to be more useful than ECP $[27,28]$. In adult asthmatics, serum EDN levels were decreased after anti IL-5 antibody treatment, indicating that this serum biomarker may represent eosinophil activation status [29]. The present study revealed that serum EDN levels had a stronger positive correlation with TEC than serum periostin did, suggesting that EDN reflects more TEC and represents the phenotypes of eo- 
sinophilic asthma.

Periostin is mainly released from airway epithelial cells stimulated by type-2 cytokines such as IL-4 and IL-13 [30], and can be used as a serum biomarker of eosinophilic airway inflammation, and to predict lung function decline and poor asthma control [31]. Because periostin is highly expressed in chronic eosinophilic inflammatory diseases, and upregulates the production of fibroblasts, periostin has an important role in airway chronic inflammation and remodeling [32]. Indeed, serum periostin is well correlated with the degree of airway eosinophilic inflammation and is suggested to be a good serologic marker for airway eosinophilia [33]. The findings of the present study indicate that serum periostin and EDN levels may be biomarkers of the phenotype of eosinophilic asthma (to determine asthmatic patient groups having sputum eosinophilia, as opposed to those with mixed granulocytic asthma). When combined, these two biomarkers can improve diagnostic discrimination. Serum EDN had more advantages with regard to reflecting TEC, while serum periostin was more effective way to represent chronic airway eosinophilic inflammation, such as airway remodeling.

Among neutrophil-dominant groups, S1ooA9 levels were higher in $\mathrm{G}_{2}$ and $\mathrm{G}_{3}$, and may be a useful serum marker to discriminate G2 from G1 plus G2. S10oA8/9 were mainly expressed on neutrophils and secreted by bronchial epithelial cells [34] and induced the activation and migration of neutrophils [35]. In the present study, SiooA9 level, not S1ooA8, differed significantly among the four groups. Although we measured other neutrophilic markers, such as IL- 8 and myeloperoxidase, no significant differences were observed among the groups (data not shown). These findings suggest that mediators secreted by epithelial cells, such as periostin and S1ooA9, have important roles in modulating inflammatory cell profiles in the asthmatic airway.

Folliculin is the causative gene of Birt-Hogg-Dube syndrome. It has a role in maintaining the integrity and function of cell-to-cell adhesion in airway epithelial cells [36]. In a recent study on toluene diisocyanate-induced asthma, folliculin production was increased by airway epithelial damage [9]. In patients with AERD, serum folliculin levels (released from human airway epithelial cells) were significantly higher than those of the aspirin-tolerant group, indicating that increased serum folliculin levels represent the status of epithelial activation and disruption [37]. In the present study, the highest folliculin level was noted in $\mathrm{G}_{4}$, and the subjects with AERD had significantly higher folliculin levels than the aspirin-tolerant subjects $(p=0.024)$. Although the pathogenic mechanisms of paucigranulocytic asthma remain poorly understood, and its characterization is less well defined, paucigranulocytic asthma is an asthma phenotype with no evidence of increased numbers of inflammatory cells. It is considered to be noninflammatory or low-grade airway inflammation and manifests as an uncoupling of airway obstruction from airway inflammation that can be driven by structural changes within the airways [20]. Taken together, epithelial cell activation and dysintegrity rather than cellular inflammation may be a major mechanism for the development of paucigranulocytic asthma, in which various triggers may alter structural cell function (myofibroblasts, epithelial, smooth muscle, and mast cells).

Major limitations of our study were that this was a cross-sectional study enrolled at a single tertiary asthma center. Although the gold standard phenotype in sputum cellular profiles has been defined as the most common finding from sputum samples taken over 5 months [38], samples collected at enrollment were used in our study. Additionally, many factors affect inflammatory cell profiles in induced sputum, such as infections, exacerbations, and environmental air pollution [39,40]. Therefore, these results can vary according to the time of sample collection and the treatment status of each patient. It is critical to find a stable serum biomarker for monitoring the various clinical states of adult asthmatic patients.

In conclusion, the serum periostin and EDN levels may be used to discriminate eosinophilic asthma from mixed granulocytic asthma. Also, the serum S10oAg level is elevated in patients with neutrophilic asthma and the serum folliculin level is elevated in those with paucigranulocytic asthma.

\section{KEY MESSAGE}

1. The cellular profile of sputum can be used to classify the phenotype of asthma, such as eosinophilic asthma, mixed granulocytic asth- 
ma, neutrophilic asthma, and paucigranulocytic asthma.

2. Serologic markers, including eosinophil-derived neurotoxin (EDN) and periostin, can be used to identify eosinophilic asthma. Additionally, the EDN level reflects blood eosinophilia and periostin indicates chronic eosinophilic inflammation of the airway.

3. The serum EDN and periostin levels can be useful markers of eosinophilic asthma and the serum S1ooAg level is indicative of neutrophilic asthma.

\section{Conflict of interest}

No potential conflict of interest relevant to this article was reported.

\section{Acknowledgments}

This study was funded by a grant of the Korea Health Technology R\&D Project through the Korean Health Industry Development Institute (KHIDI) funded by the Ministry of Health and Welfare, Republic of Korea (HI16Co992).

\section{REFERENCES}

1. Moore WC, Meyers DA, Wenzel SE, et al. Identification of asthma phenotypes using cluster analysis in the Severe Asthma Research Program. Am J Respir Crit Care Med 2010;181:315-323.

2. Katial RK, Bensch GW, Busse WW, et al. Changing paradigms in the treatment of severe asthma: the role of biologic therapies. J Allergy Clin Immunol Pract 2017;5:S1-S14.

3. Wang $\mathrm{YH}$, Voo KS, Liu B, et al. A novel subset of $\mathrm{CD}_{4}(+)$ $\mathrm{T}(\mathrm{H}) 2$ memory/effector cells that produce inflammatory IL-17 cytokine and promote the exacerbation of chronic allergic asthma. J Exp Med 2010;207:2479-2491.

4. Irvin C, Zafar I, Good J, et al. Increased frequency of dual-positive $\mathrm{TH} 2 / \mathrm{TH}_{17}$ cells in bronchoalveolar lavage fluid characterizes a population of patients with severe asthma. J Allergy Clin Immunol 2014;134:1175-1186.

5. Liu W, Liu S, Verma M, et al. Mechanism of $\mathrm{T}(\mathrm{H})_{2} /$ $\mathrm{T}(\mathrm{H}) 17$-predominant and neutrophilic $\mathrm{T}(\mathrm{H})_{2} / \mathrm{T}(\mathrm{H})_{17}$-low subtypes of asthma. J Allergy Clin Immunol 2017;139:1548-
1558.

6. Kim MA, Shin YS, Pham le D, Park HS. Adult asthma biomarkers. Curr Opin Allergy Clin Immunol 2014;14:49-54.

7. Hastie AT, Moore WC, Meyers DA, et al. Analyses of asthma severity phenotypes and inflammatory proteins in subjects stratified by sputum granulocytes. J Allergy Clin Immunol 2010;125:1028-1036.

8. Halayko AJ, Ghavami S. S1ooA8/A9: a mediator of severe asthma pathogenesis and morbidity? Can J Physiol Pharmacol 2009;87:743-755.

9. Pham DL, Trinh TH, Ban GY, Kim SH, Park HS. Epithelial folliculin is involved in airway inflammation in workers exposed to toluene diisocyanate. Exp Mol Med 2017;49:e395.

10. Davies AR, Hancox RJ. Induced sputum in asthma: diagnostic and therapeutic implications. Curr Opin Pulm Med 2013;19:60-65.

11. Katz LE, Gleich GJ, Hartley BF, Yancey SW, Ortega HG. Blood eosinophil count is a useful biomarker to identify patients with severe eosinophilic asthma. Ann Am Thorac Soc 2014;11:531-536.

12. Ullmann N, Bossley CJ, Fleming L, Silvestri M, Bush A, Saglani S. Blood eosinophil counts rarely reflect airway eosinophilia in children with severe asthma. Allergy 2013;68:402-406.

13. Lee Y, Lee JH, Yang EM, et al. Serum Levels of eosinophil-derived neurotoxin: a biomarker for asthma severity in adult asthmatics. Allergy Asthma Immunol Res 2019;11:394-405.

14. Global Initiative for Asthma. GINA report, global strategy for asthma management and prevention [Internet]. Fontana (WI): GINA, c2019 [cited 2019 Oct 2]. Available from: http://www.ginasthma.com.

15. Chung KF, Wenzel SE, Brozek JL, et al. International ERS/ ATS guidelines on definition, evaluation and treatment of severe asthma. Eur Respir J 2014;43:343-373.

16. Gibson PG, Wlodarczyk JW, Hensley MJ, et al. Epidemiological association of airway inflammation with asthma symptoms and airway hyperresponsiveness in childhood. Am J Respir Crit Care Med 1998;158:36-41.

17. Brooks CR, Gibson PG, Douwes J, Van Dalen CJ, Simpson JL. Relationship between airway neutrophilia and ageing in asthmatics and non-asthmatics. Respirology 2013;18:857-865.

18. Simpson JL, Scott R, Boyle MJ, Gibson PG. Inflammatory subtypes in asthma: assessment and identification using 
induced sputum. Respirology 2006;11:54-61.

19. ten Brinke A, Zwinderman AH, Sterk PJ, Rabe KF, Bel EH. "Refractory" eosinophilic airway inflammation in severe asthma: effect of parenteral corticosteroids. Am J Respir Crit Care Med 2004;170:601-605.

20. Tliba O, Panettieri RA Jr. Paucigranulocytic asthma: uncoupling of airway obstruction from inflammation. J Allergy Clin Immunol 2019;143:1287-1294.

21. Taylor SL, Leong LEX, Choo JM, et al. Inflammatory phenotypes in patients with severe asthma are associated with distinct airway microbiology. J Allergy Clin Immunol 2018;141:94-103.

22. Gibson PG. Inflammatory phenotypes in adult asthma: clinical applications. Clin Respir J 2009;3:198-206.

23. Shin B, Kwon HS, Park SY, Kim TB, Moon HB, Cho YS. The transition of sputum inflammatory cell profiles is variable in stable asthma patients. Asia Pac Allergy 2017;7:19-28.

24. Ntontsi P, Loukides S, Bakakos P, et al. Clinical, functional and inflammatory characteristics in patients with paucigranulocytic stable asthma: comparison with different sputum phenotypes. Allergy 2017;72:1761-1767.

25. Louis R, Lau LC, Bron AO, Roldaan AC, Radermecker M, Djukanovic R. The relationship between airways inflammation and asthma severity. Am J Respir Crit Care Med 2000;161:9-16.

26. Morioka J, Tomita M, Yoshizawa Y, Inamura H, Kurosawa M. Concentrations of eosinophil-derived neurotoxin in the blood and urine of patients with allergic diseases. Allergol Int 2004;53:359-367.

27. Kim KW, Lee KE, Kim ES, Song TW, Sohn MH, Kim KE. Serum eosinophil-derived neurotoxin (EDN) in diagnosis and evaluation of severity and bronchial hyperresponsiveness in childhood asthma. Lung 2007;185:97-103.

28. Lee YJ, Fujisawa T, Kim CK. Biomarkers for recurrent wheezing and asthma in preschool children. Allergy Asthma Immunol Res 2019;11:16-28.

29. Pham TH, Damera G, Newbold P, Ranade K. Reductions in eosinophil biomarkers by benralizumab in patients with asthma. Respir Med 2016;111:21-29.
30. Takayama G, Arima K, Kanaji T, et al. Periostin: a novel component of subepithelial fibrosis of bronchial asthma downstream of IL-4 and IL-13 signals. J Allergy Clin Immunol 2006;118:98-104.

31. Semprini R, Williams M, Semprini A, et al. Type 2 biomarkers and prediction of future exacerbations and lung function decline in adult asthma. J Allergy Clin Immunol Pract 2018;6:1982-1988.

32. O'Dwyer DN, Moore BB. The role of periostin in lung fibrosis and airway remodeling. Cell Mol Life Sci 2017;74:4305-4314.

33. Jia G, Erickson RW, Choy DF, et al. Periostin is a systemic biomarker of eosinophilic airway inflammation in asthmatic patients. J Allergy Clin Immunol 2012;130:647-654.

34. Henke MO, Renner A, Rubin BK, Gyves JI, Lorenz E, Koo JS. Up-regulation of S1ooA8 and S1ooA9 protein in bronchial epithelial cells by lipopolysaccharide. Exp Lung Res 2006;32:331-347.

35. Ryckman C, Vandal K, Rouleau P, Talbot M, Tessier PA. Proinflammatory activities of S1oo: proteins S1ooA8, S1ooA9, and S1ooA8/A9 induce neutrophil chemotaxis and adhesion. J Immunol 2003;170:3233-3242.

36. Khabibullin D, Medvetz DA, Pinilla M, et al. Folliculin regulates cell-cell adhesion, AMPK, and mTORCı in a cell-type-specific manner in lung-derived cells. Physiol Rep 2014;2:e12107.

37. Trinh HKT, Pham DL, Choi Y, Kim HM, Kim SH, Park HS. Epithelial folliculin enhances airway inflammation in aspirin-exacerbated respiratory disease. Clin Exp Allergy 2018;48:1464-1473.

38. Simpson JL, McElduff P, Gibson PG. Assessment and reproducibility of non-eosinophilic asthma using induced sputum. Respiration 2010;79:147-151.

39. Hargreave FE, Nair P. Point: is measuring sputum eosinophils useful in the management of severe asthma? Yes. Chest 2011;139:1270-1273.

40. D'silva L, Cook RJ, Allen CJ, Hargreave FE, Parameswaran K. Changing pattern of sputum cell counts during successive exacerbations of airway disease. Respir Med 2007;101:2217-2220. 